\title{
Research on Platform Lighting Remote Control System Of Taiyuan Railway Station
}

\author{
Zhao Sihai \\ School of Mechanical Electronic \& Information \\ Engineering \\ CUMTB \\ Beijing, China \\ zsh@cumtb.edu.cn \\ Liu Bo \\ School of Mechanical Electronic \& Information \\ Engineering \\ CUMTB \\ Beijing, China \\ bobbycumt@163.com
}

\author{
$\mathrm{Hu}$ Yunlong \\ School of Mechanical Electronic \& Information \\ Engineering \\ CUMTB \\ Beijing, China \\ hylong_2009@163.com
}

\begin{abstract}
Railway Platform lighting is very important to the passengers and the station's management. In Taiyuan railway station, station's staffs used to switch lighting on and off manually.It increased the difficulty of the station's management. Based on the Programmable Logic Controller (PLC) controlled, Taiyuan Railway Platform lighting remote control system changed the manual way to the remote control.Users can access the system through the IE browser. We completed hardware and software design of this railway platform lighting remote control system. Hardware design includes an analysis of the existing circuits, secondary circuit of PLC control and manual control, remote/manual switching circuit and Ethernet communication; software design includes writing the PLC control program, I/O variables connecting to the real time database, monitor screen configuration, hot standby and the WEB publishing. After we tested our system in the laboratory successfully, the system was installed the in the Taiyuan Railway Station and run stably.
\end{abstract}

Keywords-component; railway station lighting, PLC, remote control, configuration software

\section{INTRODUCTION}

Railway Platform Lighting is an important part of the station lighting system that ensures passenger safety and packaging unloading operations [1]. Lighting is located directly above the station, two railway platforms share a platform. In Taiyuan Station, platform office area and a railway platform share one platform, railway platform 2,3 share one platform, and so on. There are a total of seven railway platforms in Taiyuan Station. 4 platform lights are mounted on the platform north and south [2]. There are specialized lighting control cabinet power distribution arrangements on each platform, and there are two rooms each distribution lighting control cabinet.

Before the system transformation, Taiyuan station lighting are operated by station staff on the lighting control cabinet by switch to achieve air hoist, namely local manual control, which is direct control of the primary circuit of the switch. The primary circuit voltage is $380 \mathrm{~V}$. In order not to cause energy waste, station managers need to work on the platform staff supervision, set the reward system, which increases the difficulty of management [3]. The command center supervises all important data, such as lighting conditions, therefore, which proposed the establishment of Taiyuan station platform lighting remote control system to achieve the remote command center for lighting control.

\section{SYSTEM CONTROL REQUIREMENTS}

There are a total of seven platforms in Taiyuan station. Platform lamps provide lighting by eight lights (of which there is a platform using two lights, one light in the office area and one light in Site 1). However, the control cabinet lighting circuit is not divided according to the railway platform's number, for example, railway platforms 2,3 have two lights. The two columns of light are in accordance with the position of the canopy into the North West, North East canopy, Overhead Under the West, Under the Viaduct, Under the Elevated East, South and North West Canopy , Canopy North South East and South Canopy, Southeast Canopy for a total of nine regions. Each region is divided into two loops- two back route sites of two separately controlled lighting control cabinet, and control lights for parity complementary, so that we can ensure that if there is a period in the lighting control cabinet lighting needs an overhaul, it will not affect the site lighting.

According to the requirements of the Taiyuan railway station plant control system, it will continue to use the main circuit and PLC as the control loop, increasing core and remote control via Ethernet, providing information room server host, achieving hot standby by automatic failover, through the browser command visit system Center, and achieve an increase of air switch for manual control.

\section{HARDWARE DESIGN}

The system uses PLC as the control core, PLC needs to select the external output points by 10 points or more, and 
has Ethernet communication function, which are stable and reliable, low failure rate, and the need to take into account the costs. The system selected Siemens S7-200 series PLC CPU226CN, with 16 points relay output, the input voltage is $220 \mathrm{VAC}$, and output voltage is $24 \mathrm{VDC}$, accompanying the Ethernet module CP243-1, this module by module itself communication cables and CPU226CN connected, the power output of the power supply by CPU226CN [4]. The system will need relay outputs at 9 or 10 points, each is connected to an output point AC contactor coil. Because $\mathrm{AC}$ contactor and PLC output point is one- order and press down, the need for each AC contactor controls the circuit for positioning for a sort order configuration on the screen.

The remote server via Ethernet transceives PLC data, and in order to ensure that emergency lighting, increases the air switch for manual control, remotely switches lighting control cabinet / local control, the control object is an AC contactor coil via rotary switches, the control schematic is follows:

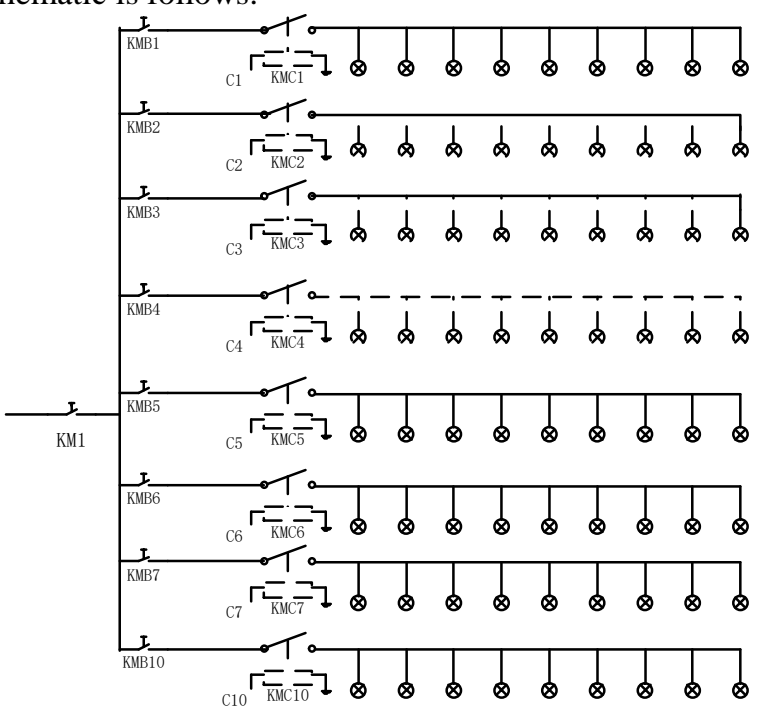

Figure 1. Primary circuitschematic diagram

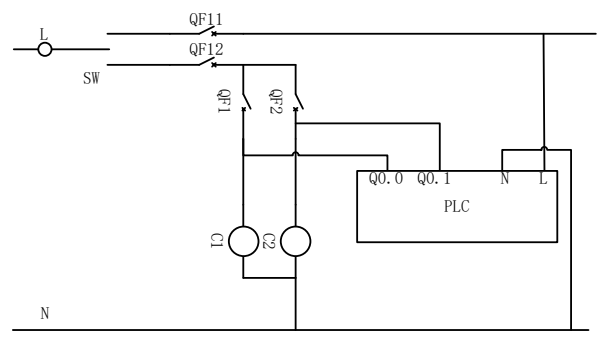

Figure 2. Secondary circuit schematic diagram

When the switch is in the "Auto" position, which is on behalf of the secondary circuit for remote control, the AC contactor coil is directly controlled by the PLC output point, and the point when the output is set to 1 , the coil is energized, and the $\mathrm{AC}$ main contact is pulled once in loop conduction; when the output point is set to 0 , the coil is energized. AC contactor main contacts open a loop that is disconnected; when switch is in the "manual" position, which is on behalf of the secondary circuit for local manual control, and the communication contactor coil is increased by new air switch control; when the air switch is closed, the corresponding coil is energized when the switch is off the air, the corresponding coil loses power.

Ethernet module CP243-1 that is connected with CPU226CN via a network cable access through fiber optic transceivers trip south elevated service switch wiring between the primary and secondary servers, have access information through the network cable host room service trip switches. Each location trip switches through clothes fiber optic connectors, which can be connected to each PLC standby server via industrial Ethernet [5-7]. System network topology is as follows:

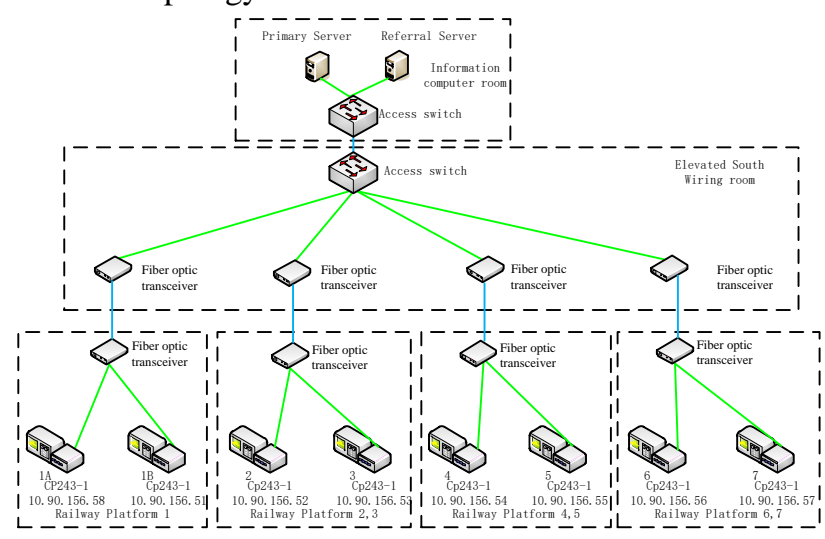

Figure 3. The topological structure of the network system diagram

\section{SYSTEM SOFTWARE DESIGN}

We must first configure and program the PLC programming software as Step7 MicroWin v4.0, the programming language for the ladder. The CPU226CN connects with USB-PPI programming cable to the PC, and PLC side using PPI port, PC side uses a USB port. To open Step7 MicroWin v4.0, you need to set the PG / PC port, the use of the programming cable to download the program, you need to select the interface PC / PPI cable. To choose a good interface, it is necessary to configure the PLC programming software, hardware, and referred to herein have CPU226CN Ethernet module CP243-1, to set the CPU in the "Communication" in, CPU226CN when connected to PC, programming software will automatically refresh the connection the device, and select the device CPU configured. In the "Ethernet Wizard" configure the Ethernet module CP243-1, the contents of the "Ethernet Wizard " in the set include the IP address, subnet mask, gateway and connection configuration, etc., in connection configuration. The PLC configured as "Server connection" to achieve PLC receives control commands and data transfer instructions issued by the remote server, it is fixed at 10.00 local TSAP property, and remote TSAP property is set to 10.11, the Ethernet module CP243-1 will be configured.

Before writing the control program, you need to define symbols for easy programming. This design of the program needs to implement the lights, turn off the lights of the function, in order to avoid the misuse of network delays that may arise, and program design with a selflocking feature, so the control of a street light program will have three variables: normally open input, often closed input and output coil. It is normally opened and normally closed inputs $\mathrm{M}$ zone variable input selection, coil output is CPU226CN Q outputs corresponding variable region, in order to facilitate the distinction, M1.0-M10.0 M district 
represents 10 lights often open input, that is, M1.1-M10.1 " lights On" function, M District 10 represents $\mathrm{NC}$ input lights, or "lights" feature, Q0.0-Q0.7, Q1.0-Q1 .1 for a total of 10 points as the output coil output. Open the symbol table, symbols are defined as follows:

TABLE I. CONTROL PROGRAM SYMBOL TABLE

\begin{tabular}{|l|l|c|c|}
\hline \multirow{2}{*}{$\begin{array}{c}\text { Serial } \\
\text { number }\end{array}$} & \multicolumn{3}{|c|}{ The Symbol Table } \\
\cline { 2 - 4 } & Symbol & Address & Notes \\
\hline 1 & K1 & M1.0 & Lighting NO.1 ON \\
\hline 2 & G1 & M1.1 & Lighting NO.1 OFF \\
\hline 3 & L1 & Q0.0 & Lighting NO.1 RUN \\
\hline
\end{tabular}

After the symbols are defined, you can call by name in the program, you do not have to remember the variable address, the program flow chart as follows:

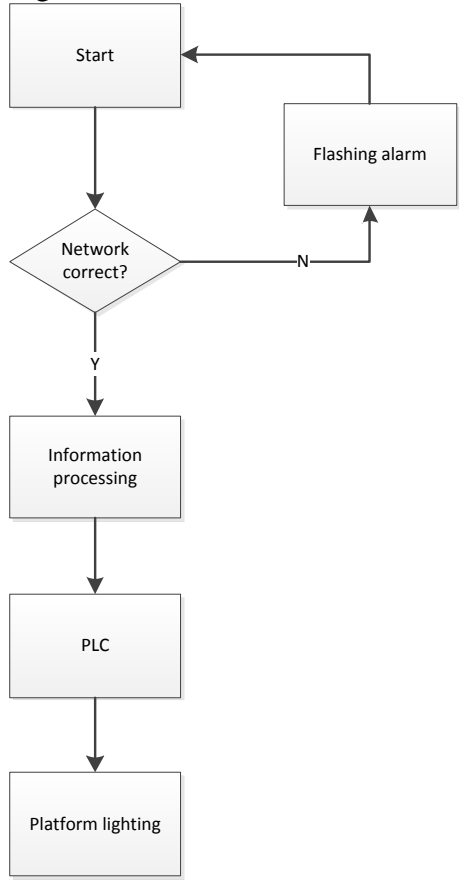

Figure 4. Program flow chart

After the program is completed, download to the PLC.

Monitoring system uses B / S mode, hot standby servers are installed in the host room and information in the command center just needs to get the monitor screen and can be accessed through a browser, and to achieve the purpose of control [8]. Configuration software selects Forcecontrol 6.1, it strength controls monitoring configuration software is the on-site production data acquisition and process control special software, the biggest feature is able for the flexible "configuration mode" rather than programming approach to system integration, it provides a good user interface and simple development project implementation. As long as the various software modules presets simple "configuration", it can be very easy to implement and monitor the completion of the functional layer. For example, in a distributed network applications, all applications (such as trend curves, alarms, etc.) a reference method for remote data and local data references are identical, the "configuration" approach can greatly reduce the automation system integration engineers' time improve the integration efficiency [9].

Create new Forcecontrol engineering project manager and $\log$ into development mode, where you need to complete the five-part work: Configure the software to connect PLC, database connection PLC variables, screen configuration, hot standby, and WEB release. (1)Configuration software needs to connect PLC IO device configuration, due to PLC via Industrial Ethernet connection configuration software, so I chose the S7200TCP SIEMENS PLC protocol IO Device Manager for device configuration. You need to configure the device name, IP address, etc. parameters, followed by eight configured PLC. (2)Database Connection PLC variables need for database configuration, database configuration is presented as in tabular form, a little table name, description, data connections, and 4 history parameter values when connecting PLC variables. Select the PLC and the PLC need to connect the point you want to connect, set up, the configuration software database point to establish a connection in the configuration software, which directly calls the database point corresponding point in the PLC that can be achieved corresponding of the point on the PLC operation. PLC variables in this article are all connected. (3)Monitor screen configuration simulate actual position lamp stand, and each is connected to a corresponding control variables to achieve control and analog displays, as follows:

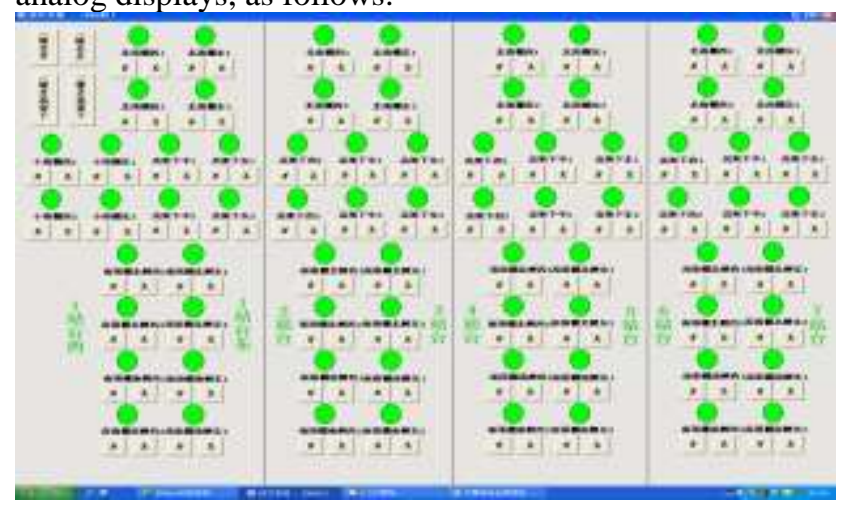

Figure 5. Monitor screen

As can be seen from Fig .5, there are 18/20 lights on each platform, which is consistent with the design of the primary circuit, each comprising four control lights , rounds, text labels and two buttons. Open circle property settings, select changing conditions in Select the Color menu, and connect corresponding variables.

In the database, 1all.PV's corresponding value is the name of the PLC output point Q0.0. 1A. When Q0.0 is true, the circle color is green; when Q0.0 is false, the circle color is red. Button's achieved function is on and off. You can set the functions via Buttons Properties, you may define the action of the left mouse movement, such as press and release the mouse button. Functionality is then realized through the scripts. lakl's corresponding database name for PLC 1A is variables M1.0, when mouse movements is pressed, M1.0 is set to 1 , when the mouse is released, M1.0 is set to 0 .

In Fig .5 also adds text labels with color, it is used to locate the position on the platform of the PLC and display the connection status, which will need to be added to the 
variables in the database. The value of the variable corresponding PLC network connection status, color, text are labeled in the blink function connection that attributes to this variable. When true, the label the repetitive red and green flashes, indicating PLC network is dropped. (4)Hot standby configuration, which is the first increase in the backup machine network node in the host system configuration, configures backup node name and the backup machine address. Dual-redundant configuration will set the unit to the host, select the newly added slave node equipment machine name. Taiyuan station in accordance with the requirements, the system operates in a host-based, backup machine only as a backup, when a host fails, the system switches to the backup machine, when the host resumes operation, the system automatically switches to the host, and this function realized the need to increase the window to run the script. Set system function ConvertTo to 1 , when the window is running, the system is forced to switch to backup status by working condition, no additional preparation machine is in this script. (5)The system uses B / S architecture. The need to carry out the project WEB publishing and in order to facilitate troubleshooting, the primary and secondary servers will be released to the local WEB. WEB server address is configured in native address. In preparation for the machine to run the same project, except that the standby machine is set up in accordance with the backup machine parameter settings.

After completing engineering, you need to set in the system settings window initial startup and initial startup program. The initial startup configuration window is set to complete the picture, when the program started, the window is automatically up and running. Initial Start program needs to check the real-time database, IO monitor, operating environment and WEB server. You can set up to start automatically and run delays, due to primary and secondary servers in the information furnished master room, you need to set the start automatically checked, in order for the network initialization time to start after Windows starts aside. The startup delay is set to 10,000 milliseconds, which is 10 seconds. The project saves the run, that we can achieve the brightest lamp control station. When the host is running, backup machine does not have control, but is able to monitor the status display. When the host is dropped, the system will automatically switch to the backup machine to running. When the host fails after repair, the system will automatically switch to running on the host. LAN PC will access control system through a browser.

\section{CONCLUSION}

After the implementation of the system is complete, we have a stable operation. Command center operators simply need to open the page to control the lighting stand, and will not cause abnormalities because of misuse operator. The addion of a key switch function at certain times provides a key to turn on and off all the lights under the doom. It also eliminates the need to manually on and off control of each road Station lamp operation, which has brought great convenience to the operator. When brigade serving in the system network fails, malfunction PLC, we could execute contingency plans, and command center staff will notify the site control cabinet lighting switch to be switched to "manual", namely local manual control. Then the control cabinet PLC power is achieved. Remote control can not be achieved to ensure the safety of the emergency state. After a period of operation, the site has been completely illuminated with remote control replacing the original local manual control.

Concurrently with the implementation of Taiyuan Station Platform Lighting System is the Taiyuan Trip Service Station Platform transformation. Lighting systems leaves connection point to the database interface, which the next time will serve to send real-time database system for train departure and arrival. After reasonable algorithms, we have achieved intelligent control platform lights under the premise of avoiding frequent system initiation [10].

\section{REFERENCES}

[1] Chen Zexin. Automatic Control System of ShangHai Railway Station PlatformLighting [J]. Chinese Railway, 2002,06:60-62.

[2] Yang Xiuqing. Automatic Power Saving Control System of Railway Passenger Station Platform Lighting [J]. Shanxi Energy Conservation , 2007,02:30-31+34

[3] Sun Jianjun. Automatic Control of Railway Platform Lighting System [J]. Resource Conservation and Environmental Protection.,2014,10:84-86

[4] Liao Changchu. S7-200 PLC Programming and Application[M] Beijing: Mechanical Industry Press, 2014

[5] Zhang Lieping, Zhang Yunsheng, Zhang Qunying. The Remote Control based on OPC and Profibus-DP Bus[J]. Control Engineering, 2008,05:594-597.

[6] Song Li. Remote Monitoring and Fault Diagnosis Based on PLC[D]. Shandong University of Science and Technology, 2004

[7] Zhao Zhenjie. Research and Implementation Based on PLC Three Level Industrial Control Network System[D]. Shandong University of Science and Technology, 2005

[8] Ma Guohua. The Application of Supervisory Control Configuration Software -- From Basic to the Practice[M]. Beijing: Chinese Electric Power Press, 2011

[9] Wu Yonggui. The Application Practice of Forcecontrol Configuration Software[M]. Beijing: Chemical Industry Press, 2013

[10] Jinlong. Transformation of Energy-saving Lighting System of Beijing West Railway Station[J]. Railway Technology Innovation, 2009,02:22-23. 